\title{
Breast MRI: EUSOBI recommendations for women's information
}

\author{
Ritse M. Mann ${ }^{1}$ - Corinne Balleyguier ${ }^{2}$ - Pascal A. Baltzer ${ }^{3}$ - Ulrich Bick $^{4}$. \\ Catherine Colin $^{5} \cdot$ Eleanor Cornford $^{6}$ • Andrew Evans ${ }^{7}$ - Eva Fallenberg ${ }^{4}$. \\ Gabor Forrai $^{8}$ - Michael H. Fuchsjäger ${ }^{9}$. Fiona J. Gilbert ${ }^{10}$ • Thomas H. Helbich $^{3}$. \\ Sylvia H. Heywang-Köbrunner ${ }^{11}$ • Julia Camps-Herrero ${ }^{12}$ • Christiane K. Kuhl ${ }^{13}$. \\ Laura Martincich $^{14}$ • Federica Pediconi ${ }^{15}$ - Pietro Panizza ${ }^{16}$ - Luis J. Pina ${ }^{17}$. \\ Ruud M. Pijnappel $^{18}$ - Katja Pinker-Domenig ${ }^{3}$ - Per Skaane ${ }^{19}$ - Francesco Sardanelli $^{20}$. \\ for the European Society of Breast Imaging (EUSOBI), with language review by Europa \\ Donna-The European Breast Cancer Coalition
}

Received: 6 February 2015 /Revised: 28 February 2015 / Accepted: 15 April 2015 /Published online: 23 May 2015

(C) The Author(s) 2015. This article is published with open access at Springerlink.com

\section{Abstract}

This paper summarizes information about breast MRI to be provided to women and referring physicians. After listing contraindications, procedure details are described, stressing the need for correct scheduling and not moving during the examination. The structured report including BI-RADS ${ }^{\circledR}$ cat-
Electronic supplementary material The online version of this article (doi:10.1007/s00330-015-3807-z) contains supplementary material, which is available to authorized users.

\section{Francesco Sardanelli}

francesco.sardanelli@unimi.it

1 Department of Radiology, Radboud University Nijmegen Medical Centre, Nijmegen, The Netherlands

2 Department of Radiology, Gustave-Roussy Institute, Villejuif, France

3 Department of Biomedical Imaging and Image-guided Therapy, Division of Molecular and Gender Imaging, Medical University of Vienna, Vienna, Austria

4 Clinic of Radiology, Charité, Universitätsmedizin Berlin, Berlin, Germany

5 Radiology Unit, Hospices Civils de Lyon, Centre HospitaloUniversitaire Lyon Sud, Pierre Bénite Cedex, France

6 Nottingham Breast Institute, Nottingham University Hospitals, Nottingham, UK

7 Dundee Cancer Centre, Clinical Research Centre, Ninewells Hospital and Medical School, Dundee, UK

8 MHEK Teaching Hospital University Semmelweis, Budapest, Hungary

9 Department of Radiology, Medical University of Graz, Graz, Austria
10 Department of Radiology, University of Cambridge, School of Clinical Medicine, Cambridge Biomedical Campus, Cambridge, UK

11 National Reference Centre Mammography, Munich, Munich, Germany

12 Department of Radiology, Hospital de la Ribera, Alzira, Valencia, Spain

13 University Hospital of Aachen, Rheinisch-Westfälische Technische Hochschule, Aachen, Germany

14 Radiology Unit, IRCCS-FPO, Candiolo, Turin, Italy

15 Department of Radiological, Oncological and Pathological Sciences, Sapienza University, Rome, Italy

16 Department of Radiology 1, Fondazione IRCCS Istituto Nazionale dei Tumori, Milan, Italy

17 Department of Radiology, Clínica Universidad de Navarra, Pamplona, Navarra, Spain

18 Department of Imaging, University Medical Centre Utrecht, Utrecht, The Netherlands

19 Department of Radiology, Oslo University Hospital Ullevaal, University of Oslo, Oslo, Norway

20 Department of Biomedical Sciences for Health, Università degli Studi di Milano, Radiology Unit, IRCCS Policlinico San Donato, Via Morandi 30, 20097 San Donato Milanese, Milan, Italy 
egories and further actions after a breast MRI examination are discussed. Breast MRI is a very sensitive modality, significantly improving screening in high-risk women. It also has a role in clinical diagnosis, problem solving, and staging, impacting on patient management. However, it is not a perfect test, and occasionally breast cancers can be missed. Therefore, clinical and other imaging findings (from mammography/ultrasound) should also be considered. Conversely, MRI may detect lesions not visible on other imaging modalities turning out to be benign (false positives). These risks should be discussed with women before a breast MRI is requested/performed. Because breast MRI drawbacks depend upon the indication for the examination, basic information for the most important breast MRI indications is presented. Seventeen notes and five frequently asked questions formulated for use as direct communication to women are provided. The text was reviewed by Europa Donna-The European Breast Cancer Coalition to ensure that it can be easily understood by women undergoing MRI.

\section{Key Points}

- Information on breast MRI concerns advantages/ disadvantages and preparation to the examination

- Claustrophobia, implantable devices, allergic predisposition, and renal function should be checked

- Before menopause, scheduling on day 7-14 of the cycle is preferred

- During the examination, it is highly important that the patient keeps still

- Availability of prior examinations improves accuracy of breast MRI interpretation

Keywords Breast - Breast cancer - Magnetic resonance imaging $\cdot$ Access to information $\cdot$ Patient advocacy

\section{Introduction}

Initial results regarding magnetic resonance imaging (MRI) of the breast were published more than 30 years ago, but clinical use started during the 1990s after the introduction of contrastenhanced (CE) protocols [1, 2]. Breast MRI is today one of the main methods for diagnosing breast diseases, together with mammography, ultrasound, and image-guided needle biopsy. It is based on the use of (a) a strong magnetic field provided by a high-quality magnet; (b) low-energy electromagnetic waves (radiofrequency waves, similar to those of radio, television, and portable phones) radiated and received by special coils (antennas) inside the magnet and positioned close to the investigated body part. MRI can well differentiate lesions and abnormalities of the breast. However, in order to diagnose or exclude a cancer, intravenous administration of a gadoliniumcontaining contrast material $(\mathrm{CM})$ is needed $[3,4]$. Injection of
$\mathrm{CM}$ is not required for evaluation of breast implant integrity. MRI does not expose the patient to potentially dangerous radiation, but other important precautions, contraindications, and potential side effects (including those regarding $\mathrm{CM}$ ) should be considered.

In terms of cancer detection, MRI outperforms (but not entirely substitutes) both mammography and ultrasound. Its valuable diagnostic performance has been confirmed by many studies. However, MRI also detects benign lesions that would otherwise have gone unnoticed, leading to additional otherwise unnecessary work-up. Costs must also be considered, as MRI is more expensive than mammography and ultrasound. The main indications for breast MRI [5-9] are listed in Table 1.

Women's information is important not only for patient awareness about advantages and disadvantages of breast MRI, but also to be prepared for the examination. Patients need to be aware of the possible benefits and risks associated with breast MRI and of potential further investigations prompted by this exam. Moreover, technical quality of breast MRI is dependent on patient compliance.

This paper is the second of a series of recommendations for women's information issued by the European Society of Breast Imaging (EUSOBI), the first focusing on mammography [10]. It is proposed to women and physicians dealing with patients for whom breast MRI is being considered. In particular, 17 notes and 5 frequently asked questions are formulated for use as direct communication to women. As many different issues are considered, single authors generally agreeing on these recommendations can have different opinions on individual statements. Finally, differences across European countries are relevant in terms of available technology, national guidelines, clinical practices, health care system, and insurance coverage. Thus, these recommendations can have different applications under local conditions.

Table 1 Indications for breast MRI

Screening of women at high risk of breast cancer

Preoperative staging of newly diagnosed breast cancer (ipsilateral and contralateral)

Evaluation of the effect of neoadjuvant chemotherapy

Evaluation of women with breast implants

Occult primary breast carcinoma (search for breast cancer in patients with metastases and negative mammography and ultrasound)

Suspected local recurrence*

Problem solving (equivocal findings at mammography/ultrasound)*

*When needle biopsy cannot be performed

Other new indications were recently proposed, such as nipple discharge [8] and evaluation of lesions with uncertain malignant potential (so-called high-risk or B3 lesions) detected at mammography or ultrasound, and needle-biopsied under their guidance [9] 


\section{Precautions/Contraindications}

An MRI system is a relatively narrow tube in which the woman lies in prone position during a breast examination for 15 to $30 \mathrm{~min}$. Patients with severe claustrophobia are unable to undergo the examination unless they accept to be psychologically/pharmacologically prepared or sedated [11]. Because of the use of magnetic fields and radiofrequency waves, the presence of non-MRI compatible intracranial ferromagnetic clips for aneurysms and iron splinters in the eyes are absolute contraindications to MRI. In cases of doubt, an Xray examination of the orbits can be performed to rule out the presence of iron splinters. Moreover, MRI is also contraindicated in patients with implanted electronic devices such as MR-unsafe pacemakers, implantable cardioverter defibrillators, and neurostimulators.

The woman should inform the radiologist or the staff personnel (technicians/nurses) if she has tattoos or permanent make-up. These may contain iron pigments, and especially when loop-shaped (like an antenna), they may heat up and cause local burns. Tissue expanders (e.g. for breast reconstructions) may not be MR-compatible. Women with intravascular stents or metal screws or plates for osteosynthesis can safely have a breast MRI 6 weeks after implantation. A list of implantable devices and precautions needed for MR imaging can be found on the Internet [12].

As stated above, breast MRI without CM cannot answer clinical questions [3, 5-7], with the evaluation of breast implant integrity as the only exception. Women with allergic predispositions or earlier allergic reactions to any $\mathrm{CM}$ have a higher risk for allergic reactions to MRI CM. Moreover, in women with very poor kidney function (estimated glomerular filtration rate lower than $30 \mathrm{ml} / \mathrm{min} \times 1.73 \mathrm{~m}^{2}$ ), contrast injection implies a real, but very low risk of a rare disease called nephrogenic systemic fibrosis [13]; CE MRI is also generally contraindicated in pregnant women, but this condition should be evaluated on a case-by-case basis [14].

Before entering the MRI room, the patient is asked to fill out a detailed questionnaire to rule out any contraindication to examination and to $\mathrm{CM}$ injection.

Note A. If you think you may be claustrophobic, you can go to the MR centre and ask to see the MR scanner to get practical information. If you are seriously claustrophobic, discuss this with the referring physician, radiologist, and personnel of the institution where MRI is scheduled. This issue should be discussed and resolved before attending the examination. The use of a simple sedative medication to relieve the symptoms might be indicated.

Note B. If you have an implantable device such as pacemakers/defibrillators, metal implants, or breast expanders, discuss this with referring physician, as these might imply that MRI could harm you and/or damage the device. In cases of doubt about contraindication, inform the radiologist and the personnel of the institution where MRI is scheduled. This issue should be discussed before the MRI takes place. If this information has not been provided in advance, inform the personnel before the examination.

Note C. If you have an important allergic predisposition (e.g. bronchial asthma) or you had allergic reactions to drugs or CMs before, discuss this with your referring physician. In cases of serious allergic symptoms, a balance between the potential advantages of MRI and the risk of allergic reactions has to be made. Where MRI has to be performed, precautions need to be taken, including the administration of corticosteroid and antihistaminic drugs prior to the investigation. In any case, consult your radiologist before the scheduled MRI date. We recommend informing the personnel of the institution where MRI is scheduled. This issue should be discussed before MRI takes place.

Note D. To avoid a risk from MRI CM in the presence of renal failure, different regulations are adopted in European countries. Your renal function can be checked using a simple blood test (performed not before 30 days from MRI) for evaluating your creatinine level and estimating the glomerular filtration rate. In any case, inform your referring physician and radiologist if you have a history of bladder or kidney disease, diabetes mellitus, cardiac or vascular disease, multiple myeloma, Waldenström disease, or if you use diuretics or non-steroidal anti-inflammatory drugs (e.g. ibuprofen/naproxen).

\section{Scheduling}

In premenopausal women, CE MRI is preferentially performed between days 7 and 14 of the menstrual cycle, when the background enhancement of the normal fibroglandular breast tissue is low, and hence abnormalities are better detected and false positives less frequent [15-19]. During the remaining days of the menstrual cycle, lesions may be masked by enhancement of the fibroglandular tissue potentially reducing the diagnostic value of the examination. If necessary, breast MRI may be performed in the third week of the menstrual cycle, taking into consideration that the results could be suboptimal. The use of oral contraceptives does not contraindicate CE MRI, but the above defined rules should be observed. Women with irregular menses (e.g., in perimenopausal phase) may undergo blood sampling for serum progesterone to determine the optimal time for breast MRI, especially if earlier examinations have been non-diagnostic due to strong glandular enhancement [20]. Premenopausal women who need only implant integrity evaluation can undergo non- 
contrast breast MRI at any time. All postmenopausal women can undergo CE MRI at any time. In fact, postmenopusal hormone replacement therapy has been recently reported to have negligible effect on parenchymal background enhancement [21]. In any case, breast MRI optimal scheduling should not substantially delay therapy planning.

Note E. If you are premenopausal and have an appointment for a screening CE MRI, check your menstrual cycle. If the exam scheduled is not between days 7 and 14 after the first day of your period, contact the centre and try to reschedule your appointment. If CE MRI has to be performed for another indication, discuss this with your radiologist: speed is sometimes more important than adequate scheduling. Be aware that an MRI examination performed outside the most suitable phase of the cycle may cause both false positives (findings suspected to be malignant which turn out to be benign) and false negatives (apparently normal or benign findings when a cancer is present). Cycle-related scheduling is not required for assessing breast implants and $\mathrm{CM}$ administration is not planned.

Note F. If you have irregular menses (e.g. perimenopausal phase) or if you have had a hysterectomy before 50 years of age, consult your radiologist to verify the need for blood sampling for serum progesterone to determine the optimal MRI scheduling.

\section{Technique/Procedure}

Breast MRI is performed using MRI scanners working at 1.5 or 3 Tesla (1.5 Tesla=15,000 Gauss).

Clear instructions and explanation regarding the procedure are provided by a technician or a nurse. After a possible interaction with the radiologist on duty and completion of questionnaires, if CM injection is needed, the woman is asked to sign a specific informed consent. Thereafter, intravenous access is obtained, placing a small plastic cannula in the cubital vein of one arm, a simple puncture comparable to that for blood sampling. During the examination, $\mathrm{CM}$ will be injected followed by a saline flush using an automated injector. The cannula will be removed after the procedure and the puncture site will be shortly compressed to stop bleeding.

The woman should keep still during the entire examination as patient movement causes most artifacts, which strongly reduce image quality and make interpretation difficult and sometimes impossible. A warm and sometimes tingling sensation can be felt in the arm of injection. This may be more extensive and be felt throughout the body. A metallic taste may occur within the mouth. A transient headache or nausea may rarely occur.

The procedure is performed with the upper body undressed and bra removed. Any clothing containing metal, jewellery, and other foreign objects must be removed. Some centres require almost complete undressing and provide disposable clothing. Dedicated breast coils are mandatory. Women are placed prone on the MRI table with each breast in the recess of the coil. A technician or a nurse positions the breasts avoiding folding of breast tissue on the edges of the coil. In some centres, slight breast compression is applied to reduce motion artifacts. Rubber ear plugs or headphones are provided to reduce the scanner noise during image acquisition. Radiologists and technicians are able to communicate with the woman during the examination. An alarm bell is provided; when it is rung by the woman, the examination will be terminated immediately and she will be removed from the magnet. Thus, the woman can be sure that if needed, she will be assisted.

When the woman is optimally positioned, table and patient are moved into the magnet, so that her breasts are in the centre of the tube: the magnetic field is most homogeneous at that position allowing for optimal image quality. The procedure is noisy, even though ear plugs/headphones attenuate noise perception. During the examination, the staff are discouraged from talking to the woman, as this frequently induces movements and should be done only when really needed. Scan sequences produce different noises and different noise levels, more relevant being those for $\mathrm{CE}$ imaging (continuous and buzzing sound), and for the so-called diffusion-weighted images (high beeping sound). When breast implant integrity has to be evaluated, dedicated scan sequences with different noises are used.

When the examination is done, the table and the woman are taken out of the scanner, and the table is lowered. The woman is then asked to sit up to remove the venous access. The procedure commonly takes 15-30 min, except when additional sequences are done for clinical purposes. The radiologist can decide to postpone the removal of the venous access for 10 $15 \mathrm{~min}$ before the patient leaves the department (see below).

Note G. During the examination, it is of paramount importance that you keep still. When the scanner acquires data (the "sequence"), you hear a relatively strong noise, reduced by the ear plugs/headphones. You may think that movements between the different sequences do not reduce image quality. However, as images acquired over time will be subtracted from each other, also movements between different scan sequences should be avoided. 


\section{After the procedure}

When the procedure is over, the woman redresses. If $\mathrm{CM}$ had been administered, outpatients may be asked to remain in the department for about $10-15$ min to check for any very rare delayed reaction to CM. Prior to reading images, image coregistration using special software is sometimes used, and the evaluation itself, including previous examinations and clinical records, also takes time. The report is usually generated within a few days, but particular cases can require a longer time. In the case of artifacts or strong enhancement of background glandular tissue in women not examined in the best phase of the menstrual cycle or with unexpected other hormonal influences, a repeat breast MRI can be required. Depending on the findings and the indication for MRI, additional investigations may be necessary.

\section{Breast MRI report and BI-RADS ${ }^{\circledR}$ categories}

Evaluation of breast MRI should be performed by a dedicated breast radiologist. The report should contain the indication for the scan, relevant clinical information, and type and dose of administered CM. In premenopausal women, the day or the week of the menstrual cycle on which MRI was performed should be stated. Techniques used should be very briefly summarized.

Reported image findings include breast density, amount of parenchymal background enhancement, and a usually structured description of relevant abnormalities, including those in the axillae or incidental findings in the imaged part of thorax and abdomen, when visible. Side and location of any breast lesions should be described. Lymph node evaluation is not a specific aim of breast MRI, but it is possible that the exam reveals unsuspected nodal metastasis.

Each report should end with a conclusion, commonly associated with a diagnostic category and recommendations. In many European countries, a structured reporting and classification system is in use. The most commonly applied system is the Breast Imaging Reporting and Data System (BI-RADS ${ }^{\circledR}$ ) developed by the American College of Radiology [22], also used with high-resolution $3 \mathrm{~T}$ systems [23].

Conclusive BI-RADS diagnostic categories are used as follows:

- $0=$ incomplete, additional imaging evaluation is needed;

- $1=$ negative, no abnormalities;

- 2 = benign findings;

- 3 = probably benign findings (short-term follow-up within 6 months recommended; needle biopsy may be performed only in special cases, such as on patient request or highrisk patients);

- 4 = suspected malignancy (needle biopsy recommended);
- 5 = highly suspected malignancy (needle biopsy recommended);

- 6 = already histologically proven cancer (typically reserved for MRI scans made for cancer staging or in the case of neoadjuvant chemotherapy).

Recommendation of needle biopsy for BI-RADS 4-5 lesions is a general rule for isolated newly diagnosed lesions. It could not be performed in the case of a lesion adjacent or close to a lesion already known to be cancer. Around $60 \%$ of lesions initially detected at MRI are identified with second-look targeted ultrasound [24], even though this rate is variable among studies. The definition of second-look comes from the common event that a lesion undetected at first-look ultrasound is detected at the second look, when the radiologist knows from MRI where to look. In that case, needle biopsy is performed under ultrasound guidance, a faster, less invasive, and cheaper procedure than MR-guided biopsy [25]. When the lesion is not detected with ultrasound and the indication for biopsy still stands, an MRguided biopsy is indicated. It takes longer than a diagnostic breast MRI, and it is a special procedure, requiring dedicated targeting and sampling equipment as well as trained personnel. In some countries it is necessary to apply for a specific reimbursement (this is a relatively new and expensive procedure).

However, in the case MR-guided biopsy cannot be performed (e.g., dedicated equipment not available; lesion site not accessible, such as those very close to the thoracic wall), computed tomography-guided biopsy or MR-guided presurgical localization may be performed.

Note $H$. When a needle biopsy is indicated for an MRdetected finding, this doesn't mean you have cancer. Up to $50-70 \%$ of MRI findings that require biopsy turn out to be benign [26]. Targeted ultrasound, re-evaluation of mammograms, targeted mammographic views, or images obtained with digital breast tomosynthesis are useful, offering the possibility of a biopsy under ultrasound or mammography guidance. Thus, if a suspicious lesion (BI-RADS 4 or 5) is MR-detected, an image-guided needle biopsy should be performed in almost all the cases. Definition of the benign nature of an MR-detected suspicious finding using only other targeted imaging modalities without biopsy is only possible in very few cases.

Note I. In the case of MRI BI-RADS 4-5, even if targeted ultrasound and above described mammographic approaches are negative, cancer cannot be excluded: an MR-guided biopsy is required. Not all centres that perform breast MRI offer MR-guided breast biopsy. However, your radiologist should be able to refer you to a centre where MR-guided biopsy can be 
performed or to opt for needle sampling under computed tomography guidance or for MR-guided presurgical localization.

BI-RADS 3 findings form a special diagnostic category [27], with a chance to be malignant below $2 \%$ [28]. However, the actual chance of an MR-detected BI-RADS 3 lesion being malignant is sometimes higher, especially in high-risk women [29]. For a BI-RADS 3 lesion, short-term follow-up is recommended instead of biopsy due to the low malignancy probability and the expected not reduced treatment efficacy for a shortly delayed diagnosis. This implies repeat MRI examinations within 6 months and potential further repeat MRI at 1 year and 2 years after initial detection. When, at MRI follow-up, an MR-detected lesion disappears, shrinks, or remains unchanged in size, and does not show any new sign of malignancy, it can be downgraded to benign (BI-RADS 2) without biopsy. However, in some cases, mostly when the patient prefers an immediate conclusion of the diagnostic pathway, a needle biopsy can be directly performed for a BI-RADS 3 lesion.

Note J. In the case of MRI BI-RADS 3 finding, you should discuss with your radiologist and/or referring physician whether watchful waiting with a follow-up breast MRI within 6 months or biopsy should be preferred. Caution is given in high-risk women: in these women a BIRADS 3 finding has a higher probability of malignancy and biopsy is more frequently performed.

\section{Sensitivity of breast MRI}

Overall sensitivity of breast MRI for breast cancer is approximately $90 \%$, which implies that $10 \%$ of cancers may be missed. Missed cancers are in general either very small or do not have enough contrast enhancement. Sensitivity for ductal carcinoma in situ (DCIS), a noninvasive lesion, possibly a precursor of invasive cancer and similarly treated, is variable; some of them, especially those with a lower pathological grade (G1) can be missed [30-32]. Occasionally, also invasive cancers can be occult at MRI. DCIS may be depicted on mammograms as a cluster of microcalcifications, even if, in some cases, MRI findings are negative. This implies that findings from clinical examination, mammography, or ultrasound, even if only probably benign, i.e. BI-RADS 3 , should be reviewed based upon negative MRI findings [33]. Generally, if a needle biopsy is correctly indicated, a negative MRI finding cannot substitute for biopsy. Of note, sensitivity also depends on technical prerequisites, clinical indication, and reader experience.
Note K. If a needle biopsy based upon palpable abnormalities or mammography/ultrasound is indicated, you should have a needle biopsy to rule out cancer. Even though highly sensitive, breast MRI is not a perfect test and should not be used as an alternative for biopsy. Needle biopsies are performed to exclude the presence of cancer; as a consequence, when a biopsy is recommended, this does not mean that you have a cancer.

\section{Breast MRI for screening}

Due to its high sensitivity, breast MRI is an excellent screening tool. In cohorts of women with a familial increased risk for breast cancer, and of women who are carriers of BRCA1, BRCA2, or other rarer gene mutations, the superior sensitivity of breast MRI compared to other breast imaging techniques has been shown [7, 34-39]. However, MRI also has a very high sensitivity for benign breast disease. This leads to additional investigations, including repeat MRI scans, targeted ultrasound, and biopsy, as stated above. This additional burden from MRI screening is greater in women with a priori lower breast cancer risk. Moreover, MRI is a relatively expensive examination, and the need for additional investigations further increases the cost. Consequently, the cost-effectiveness of MRI screening has been questioned for women who are not at increased risk [40]. Note that healthcare reimbursement of breast MRI screening is variable among countries.

Evidence for a substantial added value of MRI as a screening tool exists for women with proven BRCA1, BRCA2, or other rarer gene mutations [7, 34-39], for a proportion of women with an elevated risk based upon their family history, and for those patients who received thoracic radiotherapy before the age of 30 years [41-43]. A recent individual patientdata meta-analysis showed that for BRCA mutation carriers, the gain in sensitivity is relevant also over the age of 50 years [44]. Guidelines throughout Europe and the United States differ substantially for the risk level deserving breast MRI screening and the age for starting and ending MRI screening.

Note L. If you have multiple cases of breast and/or ovarian cancer in your family, discuss the possibility of MRI screening with your referring physician and your radiologist. There are risk assessment systems available to estimate your risk. The referring physician or your radiologist could decide to refer you to a specialized centre for risk evaluation. The results thereof can subsequently be matched to your local/national guideline. Note that healthcare reimbursement is variable among countries.

Note M. If you were treated with thoracic radiation therapy, discuss the need of MRI and mammography 
screening with your referring physician, radiation therapy specialist, and radiologist.

\section{Breast MRI for breast cancer staging}

Most breast cancers are detected due to clinical symptoms or by screening mammography. The standard way to assess suspicious lesions is with the so-called triple assessment: mammography, ultrasound, and image-guided needle biopsy. MRI is not yet involved in initial cancer detection except in those women, usually at high risk, screened with MRI. When a breast cancer is detected, MRI may be performed to assess disease extent, look for satellite lesions, and screen for other cancers either in the affected or in the contralateral breast. MRI is much better in tumour extent evaluation than either mammography or ultrasound, even though tumour size overestimation and underestimation still occur in up to $15 \%$ of patients. Although a better documentation of tumour size and extent could lead to a better tailored surgery, with a low rate of re-interventions for positive resection margins, randomized studies that evaluated the surgical outcome of preoperative MRI gave conflicting results [45-48]. In patients with invasive lobular carcinoma, a specific diffuse growing tumour type notoriously underestimated by mammography and ultrasound, a reduction of re-excisions from $18 \%$ to $11 \%$ was observed [49], although this was not statistically significant in a meta-analysis [50]. Other suggested indications are discrepancy in tumour size among different modalities (including clinical examination) that may change the treatment strategy, breast cancer found in a high-risk woman, and eligibility for partial breast irradiation [7, 51].

Preoperative MRI also detects many additional enhancing lesions unseen with mammography and ultrasound. Approximately $50 \%$ of them are cancerous (increased up to $75 \%$ in the breast harbouring an already known malignancy), indicating that pathological verification is necessary, especially when the additional lesions are distant from the already diagnosed cancer. When additional disease is detected, this logically leads to more extensive surgery. However, this must be regarded with caution. It should be understood that breast conserving surgery in breast cancer in over $40 \%$ of patients is primarily aimed at reducing disease extent rather than being completely curative [52]. This information should be presented to patients: treatment is mostly completed by radiation therapy, chemotherapy, and/or hormonal therapy. Consequently, additional MRI-detected tumour foci may be effectively treated by these adjuvant therapies. Extension of surgery indicated by MRI might, therefore, be unnecessary. So far, there is lack of evidence of improved overall or diseasefree survival due to preoperative MRI. In any case, the possible patient gain from preoperative MRI is also dependent on the experience of the radiologist reporting the MRI, the accuracy of mapping MR-detected additional tumour extent, capabilities of the treating surgeon using the results of this imaging technique and thus on the interface between radiology and surgery.

In addition, MRI detects unsuspected cancer in the contralateral breast in approximately $3 \%$ of all women with unilateral cancer as found by conventional imaging [53], even though higher rates of contralateral otherwise undetected cancers were reported [54]. Since no radiation therapy is given to the contralateral breast, the detection of unsuspected contralateral cancer may be more relevant than detection of additional ipsilateral foci. Although in most circumstances the eventual prognosis is mainly dictated by the size and grade of the largest cancer, early detection of second cancers is associated with a slight increase in survival, especially in patients below 50 years of age $[55,56]$.

Note $\mathbf{N}$. In the case of a newly diagnosed breast cancer, preoperative MRI is a possibility for improving treatment of the already diagnosed cancer and also detecting cancer in the contralateral breast. This must be balanced against a risk that more extensive unnecessary surgery may be performed (e.g. mastectomy instead of a lumpectomy) as a consequence of MRI. Your radiologist and your surgeon can discuss with you potential advantages and disadvantages of preoperative MRI considering your particular case.

\section{Breast MRI in patients with implants}

MRI is the most sensitive technique to detect breast implant ruptures when an appropriate protocol is performed [57]. This protocol includes specialized sequences without $\mathrm{CM}$ administration.

Notably, the usual reaction to breast augmentation is to form a fibrous capsule around the implant. This capsule frequently keeps the silicone in place even after an implant rupture. In fact, up to $50 \%$ of old implants are leaky 10 years after implantation [58], usually without any symptoms. Thus, screening for implant rupture is not needed [7]. In symptomatic patients, for example, those with an extracapsular rupture (i.e. with silicone outside the fibrous capsule), the leakage and spread of silicone in the breast can be very accurately depicted with MRI. MRI is able to confirm or exclude rupture when mammography or ultrasound are inconclusive. This may facilitate the decision of the surgeon to make a revision and/or to change the implants.

The presence of implants does not affect the sensitivity of MRI for breast cancer detection: other indications for CE breast MRI remain valid in the presence of implants. 
Note $O$. In the absence of symptoms, breast implants do not need to be screened for integrity with breast MRI. However, in cases of suspected rupture, MRI is the best technique to detect possible leakage.

Note P. Breast implants do not affect the sensitivity of CE MRI for new or recurrent breast cancer.

Note $Q$. If you have breast implants and a breast MRI is planned, remember to bring with you detailed information about the model/type of the implants you have. If you don't have this information, please ask the surgeon to give you these data.

\section{Evaluation of the effect of neoadjuvant chemotherapy}

In the case of advanced breast cancer, many centres adopt protocols for reduction of the mass with neoadjuvant chemotherapy before surgery. In this setting, MRI is proposed for either early prediction of response during chemotherapy [59] or for presurgical evaluation [60,61]. A baseline MRI evaluation should be performed prior to neoadjuvant chemotherapy, as MRI images cannot be compared to initial mammography or ultrasound studies. For both early response prediction and presurgical evaluation, MRI seems to be a better test than clinical breast evaluation, mammography, or ultrasound. However, women should be aware that if MRI is used to guide surgery at the end of chemotherapy, a fraction of patients (10$20 \%$ ) may have clinically relevant underestimation or overestimation of residual cancer [7].

\section{Occult primary breast carcinoma}

After the initial detection of metastases, breast cancer may be suspected, especially when axillary nodes are involved. However, in a small fraction of patients, in whom needle biopsy of lymph nodes confirms the breast origin of the disease, mammography and ultrasound are negative. This is occult primary breast cancer, accounting for up to $1 \%$ of breast cancers. In this clinical setting, MRI can identify the primary breast cancer in about two thirds of cases, allowing for breast conserving surgery $[6,7,62]$. If breast MRI is negative, immediate surgery may be avoided. In cases of axillary metastases, patients are usually treated with radiotherapy to the ipsilateral breast. Follow-up MRI can be proposed [7].

Acknowledgements The authors thank Europa Donna-The European Breast Cancer Coalition for reviewing the text to ensure that it can be easily understood by women undergoing MRI.

The scientific guarantor of this publication is Francesco Sardanelli. The authors of this manuscript declare no relationships with any companies, whose products or services may be related to the subject matter of the article. The authors state that this work has not received any funding. No statistical methods were necessary for this paper. Institutional Review Board approval was not required because the publication is a review article.

Open Access This article is distributed under the terms of the Creative Commons Attribution-NonCommercial 4.0 International License (http:// creativecommons.org/licenses/by-nc/4.0/), which permits any noncommercial use, distribution, and reproduction in any medium, provided you give appropriate credit to the original author(s) and the source, provide a link to the Creative Commons license, and indicate if changes were made.

\section{References}

1. Heywang $\mathrm{SH}$, Hahn D, Schmidt $\mathrm{H}$ et al (1986) MR imaging of the breast using gadolinium-DTPA. J Comput Assist Tomogr 10:199204

2. Kaiser WA, Zeitler E (1989) MR imaging of the breast: fast imaging sequences with and without Gd-DTPA. Preliminary observations. Radiology 170:681-686

3. Kuhl C (2007) The current status of breast MR imaging. Part I. Choice of technique, image interpretation, diagnostic accuracy, and transfer to clinical practice. Radiology 244:356-378

4. Turnbull LW (2009) Dynamic contrast-enhanced MRI in the diagnosis and management of breast cancer. NMR Biomed 22:28-39

5. Kuhl CK (2007) Current status of breast MR imaging. Part 2. Clinical applications. Radiology 244:672-691

6. Mann RM, Kuhl CK, Kinkel K, Boetes C (2008) Breast MRI: guidelines from the European Society of Breast Imaging. Eur Radiol 18:1307-1318

7. Sardanelli F, Boetes C, Borisch B et al (2010) Magnetic resonance imaging of the breast: recommendations from the EUSOMA working group. Eur J Cancer 46:1296-1316

8. Scheurlen K, Schnitzer A, Krammer J, et al (2014) Value of galactography for the diagnostic work-up of pathological nipple discharge in multimodal breast diagnostics : Part 2: a systematic review of the literature. Radiologe 54:160-166

9. Londero V, Zuiani C, Linda A et al (2012) High-risk breast lesions at imaging-guided needle biopsy: usefulness of MRI for treatment decision. AJR Am J Roentgenol 199:W240-W250

10. Sardanelli F, Helbich T, for the European Society of Breast Imaging (EUSOBI) (2012) Mammography: EUSOBI recommendations for women's information. Insights Imaging 3:7-10

11. Eshed I, Althoff CE, Hamm B, Hermann KG (2007) Claustrophobia and premature termination of magnetic resonance imaging examinations. J Magn Reson Imaging 26:401-404

12. Institute for magnetic resonance safety, education, and research. At: http://www.mrisafety.com/. Accessed on 22 Aug 2014

13. Thomsen HS, Morcos SK, Almen T et al (2013) Nephrogenic systemic fibrosis and gadolinium-based contrast media: updated ESUR Contrast Medium Safety Committee guidelines. Eur Radiol 23: 307-318

14. Cova MA, Stacul F, Quaranta R et al (2014) Radiological contrast media in the breastfeeding woman: a position paper of the Italian Society of Radiology (SIRM), the Italian Society of Paediatrics (SIP), the Italian Society of Neonatology (SIN) and the Task Force on Breastfeeding, Ministry of Health, Italy. Eur Radiol 24: 2012-2022

15. Kuhl CK, Bieling HB, Gieseke J et al (1997) Healthy premenopausal breast parenchyma in dynamic contrast-enhanced MR imaging of 
the breast: normal contrast medium enhancement and cyclicalphase dependency. Radiology 203:137-144

16. Delille JP, Slanetz PJ, Yeh ED, Kopans DB, Garrido L (2005) Physiologic changes in breast magnetic resonance imaging during the menstrual cycle: perfusion imaging, signal enhancement, and influence of the T1 relaxation time of breast tissue. Breast J 11:236241

17. Baltzer PA, Dietzel M, Vag T et al (2011) Clinical MR mammography: impact of hormonal status on background enhancement and diagnostic accuracy. Röfo 183:441-447

18. DeMartini WB, Liu F, Peacock S et al (2012) Background parenchymal enhancement on breast MRI: impact on diagnostic performance. AJR Am J Roentgenol 198:W373-W380

19. Kajihara M, Goto M, Hirayama Y et al (2013) Effect of the menstrual cycle on background parenchymal enhancement in breast MR imaging. Magn Reson Med Sci 12:39-45

20. Ellis RL (2009) Optimal timing of breast MRI examinations for premenopausal women who do not have a normal menstrual cycle. AJR Am J Roentgenol 193:1738-1740

21. Hegenscheid K, Schmidt CO, Seipel R et al (2012) Contrast enhancement kinetics of normal breast parenchyma in dynamic MR mammography: effects of menopausal status, oral contraceptives, and postmenopausal hormone therapy. Eur Radiol 22:2633-2640

22. American College of Radiology (ACR) Breast Imaging Reporting and Data System Atlas (BI-RADS Atlas). Reston, Va, U.S.A.: American College of Radiology; 2013. At: http://www.acr.org/ Quality-Safety/Resources/BIRADS/MRI. Accessed on 21 Aug 2014

23. Pinker-Domenig K, Bogner W, Gruber S et al (2012) High resolution MRI of the breast at $3 \mathrm{~T}$ : which BI-RADS ${ }^{\circledR}$ descriptors are most strongly associated with the diagnosis of breast cancer? Eur Radiol 22:322-330

24. Spick C, Baltzer PA (2014) Diagnostic utility of second-Look US for breast lesions identified at MR imaging: systematic review and meta-analysis. Radiology 273:401-409

25. Heywang-Köbrunner SH, Sinnatamby R, Consensus Group et al (2009) Interdisciplinary consensus on the uses and technique of MR-guided vacuum-assisted breast biopsy (VAB): results of a European consensus meeting. Eur J Radiol 72:289-294

26. Smith H, Chetlen AL, Schetter S et al (2014) PPV(3) of suspicious breast MRI findings. Acad Radiol 21:1553-1562

27. Comstock C, Sung JS (2013) BI-RADS 3 for magnetic resonance imaging. Magn Reson Imaging Clin N 21:561-570

28. Spick C, Szolar DH, Baltzer PA et al (2014) Rate of malignancy in MRI-detected probably benign (BI-RADS 3 ) lesions. AJR Am J Roentgenol 202:684-689

29. Liberman L, Morris EA, Benton CL, Abramson AF, Dershaw DD (2003) Probably benign lesions at breast magnetic resonance imaging: preliminary experience in high-risk women. Cancer 98:377388

30. Neubauer H, Li M, Kuehne-Heid R, Schneider A, Kaiser WA (2003) High grade and non-high grade ductal carcinoma in situ on dynamic MR mammography: characteristic findings for signal increase and morphological pattern of enhancement. Br J Radiol 76:3-12

31. Kuhl CK, Schrading S, Bieling HB et al (2007) MRI for diagnosis of pure ductal carcinoma in situ: a prospective observational study. Lancet 370:485-492

32. Facius M, Renz DM, Neubauer H et al (2007) Characteristics of ductal carcinoma in situ in magnetic resonance imaging. Clin Imaging 31:394-400

33. Dorrius MD, Pijnappel RM, der Jansen-van Weide MC, Oudkerk M (2010) Breast magnetic resonance imaging as a problem-solving modality in mammographic BI-RADS 3 lesions. Cancer Imaging 10:S54-S58
34. Saslow D, Boetes C, Burke W et al (2007) American Cancer Society guidelines for breast screening with MRI as an adjunct to mammography. CA Cancer J Clin 57:75-89

35. Lord SJ, Lei W, Craft P et al (2007) A systematic review of the effectiveness of magnetic resonance imaging (MRI) as an addition to mammography and ultrasound in screening young women at high risk of breast cancer. Eur J Cancer 43:1905-1917

36. Warner E, Messersmith H, Causer P et al (2008) Systematic review: using magnetic resonance imaging to screen women at high risk for breast cancer. Ann Intern Med 148:671-679

37. Riedl CC, Ponhold L, Flory D et al (2007) Magnetic resonance imaging of the breast improves detection of invasive cancer, preinvasive cancer, and premalignant lesions during surveillance of women at high risk for breast cancer. Clin Cancer Res 13: $6144-6152$

38. Kuhl C, Weigel S, Schrading S et al (2010) Prospective multicenter cohort study to refine management recommendations for women at elevated familial risk of breast cancer: the EVA trial. J Clin Oncol 28:1450-1457

39. Sardanelli F, Podo F, Santoro F et al (2011) Multicenter surveillance of women at high genetic breast cancer risk using mammography, ultrasonography, and contrast-enhanced magnetic resonance imaging (the high breast cancer risk italian 1 study): final results. Invest Radiol 46:94-105

40. Taneja C, Edelsberg J, Weycker D et al (2009) Cost-effectiveness of breast cancer screening with contrast-enhanced MRI in high-risk women. J Am Coll Radiol 6:171-179

41. Sung JS, Lee CH, Morris EA, Oeffinger KC, Dershaw DD (2011) Screening breast MR imaging in women with a history of chest irradiation. Radiology 259:65-71

42. Ng AK, Garber JE, Diller LR et al (2013) Prospective study of the efficacy of breast magnetic resonance imaging and mammographic screening in survivors of Hodgkin lymphoma. J Clin Oncol 31: 2282-2288

43. Tieu MT, Cigsar C, Ahmed S (2014) Breast cancer detection among young survivor of pediatric Hodgkin lymphoma with screening magnetic resonance imaging. Cancer 120:2507-2513

44. Phi XA, Houssami N, Obdeijn IM et al (2015) Magnetic resonance imaging improves breast screening Sensitivity in BRCA Mutation Carriers Age $\geq 50$ Years: Evidence From an Individual Patient Data Meta-Analysis. J Clin Oncol 33:349-356

45. Turnbull L, Brown S, Harvey I et al (2010) Comparative effectiveness of MRI in breast cancer (COMICE) trial: a randomised controlled trial. Lancet 375:563-571

46. Peters NH, van Esser S, van den Bosch MA et al (2011) Preoperative MRI and surgical management in patients with nonpalpable breast cancer: the MONET - randomised controlled trial. Eur J Cancer 47:879-886

47. Sakakibara M, Nagashima T, Sangai T et al (2008) Breastconserving surgery using projection and reproduction techniques of surgical-position breast MRI in patients with ductal carcinoma in situ of the breast. J Am Coll Surg 207:62-68

48. Gonzalez V, Sandelin K, Karlsson A et al (2014) Preoperative MRI of the breast (POMB) influences primary treatment in breast cancer: a prospective, randomized, multicenter study. World J Surg 38: 1685-1693

49. Mann RM, Loo CE, Wobbes T et al (2010) The impact of preoperative breast MRI on the re-excision rate in invasive lobular carcinoma of the breast. Breast Cancer Res Treat 119:415-422

50. Houssami N, Turner R, Morrow M (2013) Preoperative magnetic resonance imaging in breast cancer: meta-analysis of surgical outcomes. Ann Surg 257:249-255

51. Di Leo G, Trimboli RM, Benedek A et al (2015) Magnetic resonance imaging for selecting patients for partial breast irradiation: a systematic review and meta-analysis. Radiology. doi:10.1148/ radiol.2015142508 
52. Holland R, Veling SH, Mravunac M, Hendriks JH (1985) Histologic multifocality of Tis, T1-2 breast carcinomas. Implications for clinical trials of breast-conserving surgery. Cancer 56:979-990

53. Plana MN, Carreira C, Muriel A et al (2012) Magnetic resonance imaging in the preoperative assessment of patients with primary breast cancer: systematic review of diagnostic accuracy and metaanalysis. Eur Radiol 22:26-38

54. Pediconi F, Catalano C, Roselli A et al (2007) Contrast-enhanced MR mammography for evaluation of the contralateral breast in patients with diagnosed unilateral breast cancer or high-risk lesions. Radiology 243:670-680

55. Hartman M, Czene K, Reilly M et al (2007) Incidence and prognosis of synchronous and metachronous bilateral breast cancer. J Clin Oncol 25:4210-4216

56. Robertson C, Arcot Ragupathy SK, Boachie C et al (2011) The clinical effectiveness and cost-effectiveness of different surveillance mammography regimens after the treatment for primary breast cancer: systematic reviews registry database analyses and economic evaluation. Health Technol Assess 15:v-322
57. Hold PM, Alam S, Pilbrow WJ et al (2012) How should we investigate breast implant rupture? Breast J 18:253-256

58. Brown SL, Middleton MS, Berg WA, Soo MS, Pennello G (2000) Prevalence of rupture of silicone gel breast implants revealed on MR imaging in a population of women in Birmingham, Alabama. AJR Am J Roentgenol 175:1057-1064

59. Marinovich ML, Sardanelli F, Ciatto S et al (2012) Early prediction of pathologic response to neoadjuvant therapy in breast cancer: systematic review of the accuracy of MRI. Breast 21:669-677

60. Marinovich ML, Houssami N, Macaskill P et al (2013) Metaanalysis of magnetic resonance imaging in detecting residual breast cancer after neoadjuvant therapy. J Natl Cancer Inst 105:321-333

61. Marinovich ML, Macaskill P, Irwig L et al (2013) Meta-analysis of agreement between MRI and pathologic breast tumour size after neoadjuvant chemotherapy. Br J Cancer 109:1528-1536

62. Lu H, Xu YL, Zhang SP et al (2011) Breast magnetic resonance imaging in patients with occult breast carcinoma: evaluation on feasibility and correlation with histopathological findings. Chin Med J (Engl) 124:1790-1795 